\title{
LETRAMENTO EM SAÚDE E FATORES ASSOCIADOS EM ADULTOS USUÁRIOS DA ATENÇÃO PRIMÁRIA
}

\author{
HEALTH LITERACY AND ASSOCIATED FACTORS IN \\ ADULTS PRIMARY CARE USERS
}

\author{
ALFABETIZACIÓN EN SALUD Y FACTORES ASOCIADOS EN \\ ADULTOS USUARIOS DE LA ATENCIÓN PRIMARIA
}

Suzana Raquel Lopes Marques ${ }^{1}$

Stela Maris Aguiar Lemos ${ }^{2}$

Resumo O objetivo da pesquisa foi investigar o letramento em saúde e associação com fatores sociodemográficos, autopercepção da saúde e qualidade de vida em adultos. Tratou-se de estudo transversal com adultos entre 20 e 59 anos, usuários da atenção primária à saúde, realizado em unidades básicas de saúde, de fevereiro a maio de 2015 - amostra probabilística. O letramento em saúde foi avaliado pelo Short Assessment of Health Literacy for Portuguese-Speaking Adults. Realizou-se análise estatística descritiva, análise bivariada $(\mathrm{p} \leq 0,20)$ e regressão logística múltipla ( $\mathrm{p} \leq 0,05)$. O letramento em saúde inadequado foi frequente e inicialmente associado a sexo, escolaridade, última série cursada com aprovação, estudo formal mínimo, Critério de Classificação Econômica Brasil, plano de saúde, arranjo familiar, importância atribuída à saúde, nota atribuída à própria saúde, frequência de comparecimento à unidade básica de saúde e aos domínios relações sociais e ambiental do teste World Health Organization Quality of Life. No modelo final da regressão logística, somente a escolaridade permaneceu associada ao letramento em saúde, e indivíduos com menor escolaridade tiveram mais chance de ter letramento em saúde inadequado. Os achados sugerem a necessidade do desenvolvimento de estratégias de educação em saúde para os adultos usuários da atenção primária.

Palavras-chave alfabetização em saúde; condições sociais; educação em saúde; saúde do adulto; sistema único de saúde.
Abstract The aim was to investigate health literacy and association with social and demographic factors, self-perception of health and quality of life in adults. This is a cross-sectional study with adults between 20 and 59 years, users of primary health care, conducted in the basic health units, from February to May of 2015 - probability sample. Health literacy was evaluated by means of Short Assessment of Health Literacy for Portuguese-speaking Adults. Descriptive statistical analysis, bivariate analysis $(\mathrm{p} \leq 0.20)$ and multiple logistic regression ( $p \leq 0.05)$ were performed. Inadequate health literacy was recurrent and initially associated with sex, schooling, last grade concluded with approval, minimum formal study, Brasil Economic Classification Criterion, health insurance, family arrangement, importance attributed to health, grade attributed to own health, attendance to the basic health unit and two domains of the test World Health Organization Quality of Life (social relations and environmental). In the final model of logistic regression, only schooling remained associated with health literacy, in a way that individuals with less schooling had more chance of having inadequate health literacy. The findings suggest the need to develop health education strategies for adult users of primary care.

Keywords health literacy; social conditions; health education; adult health; unified health system. 


\section{Introdução}

A atenção primária à saúde (APS) tem papel fundamental para o bom desempenho do Sistema Único de Saúde (SUS), especialmente quando estruturada pela Estratégia Saúde da Família (ESF). Esse nível de atenção configura-se como elemento-chave pela capacidade de influir nos indicadores de saúde, função reguladora da utilização dos recursos e ordenadora do cuidado longitudinal em saúde (Rasella et al., 2014; Macinko e Harris, 2015). A longitudinalidade da assistência é considerada característica central da APS e apresenta três dimensões: a identificação da unidade básica de saúde (UBS) como fonte regular de cuidado, o estabelecimento de vínculo terapêutico duradouro entre os pacientes e os profissionais de saúde e, por fim, a continuidade informacional (registros de qualidade, que favoreçam o crescente conhecimento sobre o paciente por parte da equipe de saúde) (Cunha e Giovanella, 2011).

Na APS, o cuidado e o acompanhamento longitudinais perpassam por ações de promoção do bem-estar e prevenção de doenças/agravos (Rasella et al., 2014). Para que essas intervenções sejam bem-sucedidas, é preciso aperfeiçoar a comunicação entre o sistema de saúde, seus trabalhadores e usuários (Passamai et al., 2012). Nesse contexto, é essencial refletir sobre como as pessoas compreendem e utilizam as orientações da equipe profissional para tomar decisões e agir no cuidado consigo mesmas (Sampaio et al., 2015). Essas questões envolvem a temática do letramento em saúde, sendo necessário desenvolver estudos com usuários do SUS a esse respeito (Coelho et al., 2014).

O letramento em saúde (LS) refere-se ao

conhecimento, motivação e competências das pessoas para ter acesso, compreender, avaliar e aplicar informações de saúde a fim de fazer julgamentos e tomar decisões na vida cotidiana relativas ao autocuidado, prevenção de doenças e promoção da saúde para manter ou melhorar a qualidade de vida (World Health Organization, 2013, p.4).

O letramento em saúde pode ser classificado como básico/funcional; comunicativo/interativo e crítico. Esses diferentes níveis representam habilidades progressivas do indivíduo para compreender as questões de saúde e exercer maior controle sobre elas (Nutbeam, 2000). Esses conceitos evidenciam que o importante não é apenas saber se o indivíduo domina a leitura e a escrita, mas o que ele é capaz de fazer com essas habilidades, especificamente no âmbito da saúde. Em especial no gerenciamento de doenças crônicas (cada vez mais prevalentes como causa de morbimortalidade no Brasil), observa-se que mesmo pessoas com bom nível instrucional podem apresentar dificuldades em compreender orientações sobre os cuidados com a saúde (Sampaio et al., 2015). 
As limitações quanto ao letramento funcional em saúde dificultam a promoção e a educação em saúde (Coelho et al., 2014) e devem ser foco da atenção de profissionais da área e gestores (Ruiz et al., 2013; Beauchamp et al., 2015; Yin et al., 2015). O LS inadequado está associado a comportamentos de risco, redução do autocuidado e aumento de hospitalizações e custos (World Health Organization, 2013; Ruiz et al., 2013). O baixo letramento em saúde oral, relativo à comunicação verbal entre pacientes e profissionais de saúde, também está associado a piores resultados de saúde e deve ser pesquisado (Nouri e Rudd, 2015). Em contrapartida, fortalecer o LS tem demonstrado favorecer a resiliência individual e comunitária, auxilia no enfrentamento das iniquidades e melhora a saúde e o bem-estar (World Health Organization, 2013; Ruiz et al., 2013).

O letramento em saúde deve ser considerado como um importante resultado sensível à educação em saúde, ou seja, é mensurável e interligado a resultados mais distais em saúde (Yin et al., 2015). No contexto brasileiro, os estudos estão em fase de conhecer o LS de diferentes grupos populacionais. Essa etapa é necessária para que a literatura científica nacional se dedique ao desenvolvimento de intervenções para aprimorar o letramento em saúde (Sampaio et al., 2015).

Outro aspecto relevante no contexto da APS é a autopercepção da saúde, a qual favorece a compreensão da perspectiva do paciente sobre o próprio bem-estar e determina os comportamentos e cuidados assumidos para este fim. A autopercepção da saúde se relaciona a fatores biológicos, socioeconômicos e de vínculo com as equipes profissionais em adultos brasileiros (Agostinho et al., 2010). Sua utilização como indicador contribui para a prática clínica, como acompanhamento da evolução de saúde dos pacientes, para as pesquisas (Agostinho et al., 2010), na percepção integrada do indivíduo quanto às dimensões biológicas, psicológicas e sociais (Reichert, Loch e Capilheira, 2012), e deve ser considerada no desenvolvimento de políticas de saúde (Confortin et al., 2015).

A avaliação da qualidade de vida é também um fator importante ao contribuir para as ações de promoção à saúde na ESF, favorecendo o vínculo e o cuidado longitudinal (Chiesa et al., 2011). A qualidade de vida refere-se à “percepção do indivíduo de sua posição na vida no contexto da cultura e sistemas de valores nos quais vive e em relação aos seus objetivos, expectativas, padrões e preocupações" (World Health Organization, 2012, p.3). A literatura evidencia associação entre fatores sociodemográficos, como escolaridade, sexo e faixa etária, e qualidade de vida (Azevedo, Friche e Lemos, 2012).

Cabe ressaltar a necessidade de maior enfoque na população adulta ao se realizarem pesquisas e planejar ações em saúde (Aquino et al., 2012; Dilélio et al., 2015). Adultos apresentam perfis de morbidade e mortalidade variáveis conforme fatores biológicos, sociais, demográficos e econômicos (Aquino 
et al., 2012; Kessaram et al., 2015). Inquérito nacional verificou limitações quanto ao acesso e à continuidade dos serviços de saúde para adultos em áreas urbanas no Brasil, sendo imperativo o enfrentamento dessa realidade (Dilélio et al., 2015).

Conforme demonstrado pela literatura, o letramento em saúde deve ser considerado no planejamento e no desenvolvimento de ações em saúde (Coelho et al., 2014; Ruiz et al., 2013; Beauchamp et al., 2015; Yin et al., 2015). Fato semelhante ocorre em relação aos determinantes sociais da saúde (Paim et al., 2011), à autopercepção da saúde (Confortin et al., 2015) e à qualidade de vida (Chiesa et al., 2011). Diante desses vários aspectos que perpassam o cotidiano de usuários, profissionais e gestores no contexto da APS, acredita-se que a investigação das possíveis associações entre eles pode contribuir para o conhecimento científico e a prática clínica em saúde, assim como auxiliar a elaboração de políticas públicas mais adequadas às necessidades da sociedade.

O objetivo do estudo aqui apresentado foi investigar o letramento em saúde e a associação com fatores sociodemográficos, autopercepção da saúde e qualidade de vida em adultos usuários da atenção primária à saúde.

\section{Critérios e instrumentos para avaliação do letramento em saúde e das variáveis explicativas}

Tratou-se de estudo ${ }^{3}$ observacional analítico transversal com adultos entre 20 e 59 anos, homens e mulheres, usuários da atenção primária à saúde de município da Região Metropolitana de Belo Horizonte (RMBH), Minas Gerais, Brasil.

A pesquisa foi realizada nas unidades básicas de saúde (UBSs) do município, as quais totalizavam 16 unidades, atendendo 41.993 usuários entre 20 e 59 anos em 2013. A amostra foi probabilística, estratificada segundo a distribuição de usuários por UBS, sexo e faixa etária $(20$ a 39, 40 a 49 e 50 a 59 anos). O cálculo amostral foi definido por amostragem estratificada aleatória simples. Ao se considerarem margem de erro amostral e nível de significância de 5\%, verificou-se o tamanho da amostra necessário de 380 usuários da atenção primária à saúde, sendo o tamanho final da amostra de 380 participantes.

A faixa etária de 20 a 59 anos foi a selecionada para o estudo por se referir à faixa adulta segundo os dados populacionais informados pela Secretaria de Saúde do município analisado, os quais foram utilizados para realizar o cálculo amostral. Além disso, a literatura ressalta a lacuna existente quanto a estudos dedicados à população adulta (Aquino et al., 2012; Dilélio et al., 2015), e outras pesquisas já utilizaram faixa etária semelhante (19 a 59 anos) no contexto do letramento em saúde de adultos brasileiros (Sampaio et al., 2015; Coelho et al., 2014). 
Foram considerados critérios de inclusão: ser usuário da atenção primária à saúde via SUS no município pesquisado; ter idade entre 20 e 59 anos; ter concordado em participar do estudo e assinado o termo de consentimento livre e esclarecido. Os critérios de exclusão foram: manifestação de alterações neurológicas ou cognitivas que comprometessem a compreensão por parte do usuário dos instrumentos do estudo; e manifestação de comprometimentos relativos à expressão verbal que impossibilitassem à entrevistadora compreender as respostas apresentadas pelo usuário.

A variável resposta foi o letramento em saúde, aferida por meio da utilização do Short Assessment of Health Literacy for Portuguese-Speaking Adults (SAHLPA-184) (Apolinario et al., 2012). Trata-se de um instrumento para estimar o nível de alfabetismo em saúde, cujas propriedades psicométricas foram testadas e validadas em uma amostra de adultos brasileiros. Avalia as habilidades de pronúncia e compreensão de 18 termos médicos comuns. A aplicação e a análise do instrumento foram realizadas conforme os critérios propostos na literatura (Apolinario et al., 2012). O escore final do SAHLPA-18 é calculado consoante o indicado pelos autores do instrumento, de modo que cada item correto recebe um ponto e o escore total é obtido pela soma dos itens, variando de 0 a 18. Os itens são considerados corretos apenas quando o avaliado acerta a pronúncia e a compreensão. Um escore de 0 a 14 sugere alfabetismo em saúde inadequado; e de 15 a 18, alfabetismo em saúde adequado (Apolinario et al., 2012). Uma vez que no estudo aqui apresentado utilizou-se o termo 'letramento em saúde' (em razão de considerá-lo mais abrangente e apropriado que a terminologia 'alfabetismo em saúde'), o escore final foi considerado como letramento em saúde adequado e inadequado, mantendo-se a forma dicotômica e valores de corte propostos na literatura (Apolinario et al., 2012).

O SAHLPA foi adaptado do Short Assessment of Health Literacy for Spanish-Speaking Adults (SALHSA). Este, por sua vez, originou-se de um dos instrumentos mais utilizados para avaliação do letramento em saúde, o Rapid Estimate of Adult Literacy in Medicine (REALM). Portanto, apesar de as propriedades psicométricas do SAHLPA-18 terem sido avaliadas em pessoas com idade $\geq 60$ anos, ressalta-se que no instrumento que o originou, SAHLSA (Lee et al., 2010), a amostra analisada incluiu adultos (de 18 a menores de 80 anos). Assim, ao se considerar a origem do instrumento SAHLPA-18, é possível constatar sua adequação à avaliação de adultos realizada no estudo que deu origem a este artigo.

As variáveis explicativas referiram-se a aspectos sociodemográficos, de autopercepção da saúde e de qualidade de vida. Já as variáveis referentes aos fatores sociodemográficos foram aferidas por meio do questionário sociodemográfico e do Critério de Classificação Econômica Brasil (CCEB) (Associação Brasileira de Empresas de Pesquisa, 2012). O questionário sociodemográfico 
foi composto pelos eixos: identificação; moradia e estrutura familiar; escolaridade; trabalho e acesso à rede de saúde particular. No eixo identificação, a idade dos participantes foi coletada de forma contínua; no eixo escolaridade, para favorecer a triangulação dos resultados, os dados foram coletados tendo como referência três maneiras distintas: categórica, por nível de ensino, inicialmente em sete categorias (analfabeto, ensino fundamental incompleto, ensino fundamental completo, ensino médio incompleto, ensino médio completo, ensino superior incompleto, ensino superior completo); contínua, pelo relato de anos de estudo formal; e contínua, pelo relato da última série cursada com aprovação. Para análise estatística dos anos de estudo formal, calculou-se a mediana tomando-se como base os dados contínuos referentes a essa variável.

As variáveis acerca da autopercepção de saúde foram avaliadas por meio do questionário de autopercepção da saúde. O instrumento foi elaborado pelas autoras e composto por eixos temáticos: percepção da própria saúde (importância atribuída à saúde, frequência na qual o participante pensa na própria saúde e nos problemas de saúde; percepção de comprometimento das atividades cotidianas devido ao estado de saúde atual; percepção de si como pessoa saudável); autopercepção da saúde (pergunta 'Como você avalia a sua saúde?', com resposta em escala Likert de cinco pontos - atribuição de uma nota para a própria saúde, em escore de zero a dez, em números inteiros, sendo zero referente a saúde muito ruim e dez, a excelente); aspectos de morbidade (presença e número de problemas de saúde, segundo o relato dos participantes) e utilização dos serviços de saúde (motivo de ter ido à UBS no dia da entrevista, frequência de comparecimento à UBS e tempo decorrido desde o último comparecimento).

Por fim, as variáveis relativas à qualidade de vida foram avaliadas por meio do teste World Health Organization Quality of Life (WHOQOL abreviado), versão em português (Fleck et al., 2000). Esse instrumento é constituído por 26 questões, com respostas estruturadas em escala tipo Likert. As duas primeiras questões são analisadas separadamente e referem-se, respectivamente, à percepção geral do indivíduo sobre a própria qualidade de vida e sobre o quão satisfeito está com a própria saúde. As demais 24 questões abordam os domínios físico, psicológico, das relações sociais e do meio ambiente. Esses quatro domínios possibilitam a avaliação individual da qualidade de vida em diversos grupos e situações, em populações saudáveis ou acometidas por doenças crônicas (Fleck et al., 2000; Kluthcovsky e Kluthcovsky, 2009).

Uma vez que a consistência interna do WHOQOL abreviado foi avaliada como boa, quer sejam consideradas as 26 questões, quer sejam os quatro domínios ou cada um dos domínios no teste (Fleck et al., 2000), no estudo aqui apresentado optou-se por avaliar a qualidade de vida mediante a análise dos quatro domínios do WHOQOL abreviado. 
Estruturou-se uma base de dados no software Microsoft Office Excel 2013 com as respostas coletadas. Então utilizou-se uma ferramenta desenvolvida para realizar o cálculo do escore e estatística descritiva do instrumento, seguindo a sintaxe proposta pelo Grupo WHOQOL (Pedroso et al., 2010). Os escores dos quatro domínios do WHOQOL são apresentados em uma escala de 0 a 100, cujos resultados mais próximos ao valor 100 sugerem melhor qualidade de vida relativa a cada domínio do teste (Pedroso et al., 2010).

O recrutamento e a coleta de dados foram realizados de fevereiro a maio de 2015, nas UBSs de referência de cada participante no momento de espera para o atendimento de saúde.

Realizou-se estudo-piloto prévio com cinco usuários, em uma das UBSs, para ajuste da terminologia e calibração dos instrumentos. O estudo-piloto possibilitou também a prática da abordagem a ser realizada para convidar e aplicar os instrumentos de avaliação, bem como a estimativa do tempo de cada entrevista.

Quanto à análise dos dados, realizou-se análise descritiva por meio da distribuição de frequência para as variáveis categóricas e das medidas de tendência central e de dispersão para as variáveis contínuas. Para tanto, os dados foram previamente digitados em base de dados elaborada no software Microsoft Office Excel 2013 e conferidos.

Posteriormente, foi realizada análise bivariada para verificar a associação entre as variáveis resposta e explicativas. Para avaliar a associação com variáveis categóricas, utilizou-se o teste qui-quadrado de Pearson. Uma vez que todas as variáveis contínuas tiveram distribuição assimétrica pelo teste de Kolmogorov-Smirnof ( $\mathrm{p}<0,005)$, foi utilizado o teste não paramétrico de Mann Whitney para comparação entre as medianas dos dois grupos da variável-resposta (Siqueira e Tibúrcio, 2011).

Algumas das variáveis explicativas foram reclassificadas para reduzir as categorias com poucas informações e melhorar as estimativas nas análises: - Escolaridade: foi reorganizada nas categorias ensino fundamental (referente ao ensino fundamental completo e incompleto), ensino médio (médio completo e incompleto) e ensino superior (superior e pós-graduação completo e incompleto). Considerou-se mais adequado realizar essa reclassificação de modo a agregar os níveis de ensino incompletos aos completos por ponderar que esta seria a maneira mais coerente de realizar a análise estatística - e que levaria em consideração as competências escolares adquiridas pelos participantes que cursaram, mesmo que parcialmente, cada nível de ensino.

- Situação ocupacional: foram mantidas as categorias empregado, autônomo, desempregado, do lar e acrescida a categoria outros (afastados por motivo de saúde, aposentados e estudantes). 
- Situação conjugal: foi mantida a categoria solteiro e foram unificados os casados/união estável, bem como os separados/divorciados/viúvos.

- CCEB: as categorias foram reorganizadas em A e B (A1, A2, B1 e B2), C (Cl e C2), D e E (D e E).

- Situação de moradia: foi mantida a categoria própria e criada a categoria alugada/cedida (alugada/cedida/compartilhada com herdeiros/área de ocupação).

- Importância atribuída à saúde: foram unificadas as categorias sem importância/pouco importante/indiferente e mantidas as categorias importante e muito importante.

- Avaliação da própria saúde: foram utilizadas as categorias ruim (muito ruim/ruim/nem ruim, nem boa) e boa (boa/muito boa).

- Frequência em que pensa na saúde/problemas de saúde e frequência de comparecimento à UBS: foram unificadas as categorias nunca/raramente e mantidas as categorias às vezes, frequentemente e sempre.

A análise bivariada foi realizada com os 368 participantes que responderam ao instrumento de avaliação do letramento em saúde (SAHLPA-18) de forma completa.

As variáveis com associações estatisticamente significantes ao nível de $20 \%$ ( $\mathrm{p} \leq 0,20)$ na análise bivariada foram consideradas para entrada no modelo de regressão logística múltipla, com exceção da variável 'último ano cursado' e 'estudo formal mínimo' por serem altamente relacionadas à variável 'escolaridade', que foi escolhida para essa análise. Adotou-se o método manual, considerando em cada passo da análise a variável com maior valor-p para retirada do modelo. Posteriormente à análise bivariada, no modelo final da regressão logística, foram consideradas como variáveis com associação significante aquelas ao nível de $5 \%(\mathrm{p} \leq 0,05)$. A magnitude das associações foi avaliada pela odds ratio (OR) e seus respectivos intervalos de confiança a 95\%. A adequação do modelo foi avaliada pelo teste de Hosmer e Lemeshow (Siqueira e Tibúrcio, 2011). Os modelos de regressão logística foram ajustados com as seguintes variáveis: sexo, escolaridade, $\mathrm{CCEB}$, plano de saúde, arranjo familiar, importância da saúde, frequência de comparecimento à UBS; nota da saúde e domínios do WHOQOL que apresentaram valor-p<0,20.

Para entrada, processamento e análise dos dados quantitativos foi utilizado o programa Statistical Package for the Social Sciences, versão 21.0.

Previamente à coleta de dados, foi solicitada e obtida autorização da Secretaria Municipal de Saúde e da gerência de cada UBS por meio de carta de anuência acompanhada de cópia do projeto de pesquisa. O estudo que originou este artigo foi submetido ao Comitê de Ética em Pesquisa da Universidade Federal de Minas Gerais e aprovado com o n. Certificado de Apresentação para Apreciação Ética 25014513.7.0000.5149. 


\section{Letramento em saúde e as variáveis explicativas}

Quanto às questões sociodemográficas, no eixo temático identificação verificou-se que a maioria dos 380 participantes era do sexo feminino $(51,6 \%)$, mediana de idade de 37,0 (desvio padrão - DP 11,0) anos, sendo o local de nascimento mais frequente o próprio município no qual o estudo se realizou $(56,6 \%)$. Nascidos em outros municípios do estado de Minas Gerais totalizaram $34,5 \%$, e em outros estados da federação, 8,9\%. No que se refere ao eixo moradia e estrutura familiar, $67,5 \%$ dos entrevistados residiam em casa própria; predominou a situação conjugal casado ou em união estável $(58,2 \%)$, e em média 4,0 (DP 1,7) pessoas residiam na mesma moradia. No eixo escolaridade, a mediana de anos de estudo formal foi de 11,0 (DP 3,8). O nível de ensino formal relatado foi nenhum para $1,3 \%$ dos entrevistados; ensino fundamental incompleto ou completo para 42,3\%; ensino médio incompleto ou completo para 44,8\%; e ensino superior ou pós-graduação incompleto ou completo para $11,5 \%$. No eixo trabalho, as situações ocupacionais mais prevalentes foram: empregado $(46,1 \%)$, autônomo $(22,9 \%)$, do lar $(12,4 \%)$ e desempregado (10,0\%); os demais participantes relataram ser estudantes, aposentados ou afastados por motivo de saúde $(8,7 \%)$. Quanto às atividades ocupacionais, a maioria dos 380 participantes relatou ser trabalhador dos serviços, vendedor do comércio em lojas e mercados $(31,6 \%)$; trabalhador da produção de bens e serviços industriais $(18,9 \%)$ e técnico de nível médio $(6,1 \%)$. No eixo acesso à rede de saúde particular, observou-se que a maioria dos participantes $(68,9 \%)$ não tinha plano de saúde. Dentre os $31,1 \%$ que dispunham de plano de saúde, a duração do contrato com a rede particular apresentou mediana de 4,0 (DP 6,6) anos. Quanto à classificação econômica, dos 372 participantes que responderam integralmente ao CCEB, 33,9\% compunham as classes A e B, 56,7\% a classe C e 9,4\% as classes D e E.

A Tabela 1 apresenta a distribuição de frequências de variáveis do questionário de autopercepção da saúde. Destaca-se que a maioria dos entrevistados considerava a própria saúde como importante/muito importante $(97,6 \%)$, pensava na saúde e nos problemas de saúde frequentemente/sempre (respectivamente, $71,3 \%$ e $64,3 \%$ ), considerava-se saudável $(80,1 \%)$ e avaliava a própria saúde como boa/muito boa $(83,9 \%)$. Contudo, mais da metade dos adultos analisados $(53,4 \%)$ referiu-se à presença de problemas de saúde. A frequência de comparecimento à UBS foi bastante variável, sendo mais recorrente o comparecimento raro $(40,3 \%)$ e trimestral/semestral $(32,2 \%)$. 
Tabela 1

\begin{tabular}{|c|c|c|}
\hline \multicolumn{3}{|c|}{ Percepção da própria saúde } \\
\hline Importância da saúde & $\mathbf{n}$ & $\%$ \\
\hline Sem importância/Pouco importante/Indiferente & 9 & 2,4 \\
\hline Importante/Muito importante & 371 & 97,6 \\
\hline Total & 380 & 100,0 \\
\hline Frequência em que pensa na saúde & $\mathbf{n}$ & $\%$ \\
\hline Nunca/Raramente/Às vezes & 109 & 28,7 \\
\hline Frequentemente/Sempre & 271 & 71,3 \\
\hline Total & 380 & 100,0 \\
\hline Frequência em que pensa nos problemas de saúde & $\mathbf{n}$ & $\%$ \\
\hline Nunca/Raramente/Às vezes & 135 & 35,7 \\
\hline Frequentemente/Sempre & 243 & 64,3 \\
\hline Total & 378 & 100,0 \\
\hline $\begin{array}{l}\text { Frequência em que o estado de saúde atual compro } \\
\text { cotidianas }\end{array}$ & $\mathbf{n}$ & $\%$ \\
\hline Nunca/Raramente/Às vezes & 330 & 87,1 \\
\hline Frequentemente/Sempre & 49 & 12,9 \\
\hline Total & 379 & 100,0 \\
\hline Considera-se saudável & $\mathbf{n}$ & $\%$ \\
\hline Sim & 301 & 80,1 \\
\hline Não & 75 & 19,9 \\
\hline Total & 376 & 100,0 \\
\hline \multicolumn{3}{|c|}{ Autopercepção da saúde } \\
\hline Como avalia a própria saúde & $\mathbf{n}$ & $\%$ \\
\hline Muito ruim/Ruim/Nem ruim, nem boa & 61 & 16,1 \\
\hline Boa/Muito boa & 319 & 83,9 \\
\hline Total & 380 & 100,0 \\
\hline \multicolumn{3}{|c|}{ Aspectos de morbidade } \\
\hline Relato de presença de problema de saúde & $\mathbf{n}$ & $\%$ \\
\hline Sim & 203 & 53,4 \\
\hline Não & 177 & 46,6 \\
\hline Total & 380 & 100,0 \\
\hline \multicolumn{3}{|c|}{ Utilização dos serviços de saúde } \\
\hline Frequência de comparecimento à UBS & $\mathbf{n}$ & $\%$ \\
\hline Mais de uma vez por semana/Semanalmente & 35 & 9,3 \\
\hline Mensalmente & 69 & 18,2 \\
\hline Trimestralmente/Semestralmente & 122 & 32,2 \\
\hline Raramente & 153 & 40,3 \\
\hline Total & 379 & 100,0 \\
\hline
\end{tabular}

Fonte: As autoras.

Nota: *O número de informações varia devido a dados faltantes.

UBS: unidade básica de saúde 
No eixo temático autopercepção da saúde, a média da nota atribuída à própria saúde foi 8,0 (DP 1,6). Em relação ao eixo aspectos de morbidade, verificou-se que os problemas de saúde mais frequentemente referidos $(\mathrm{n}=380)$ eram os do aparelho circulatório $(25,8 \%)$; endócrinos, nutricionais e metabólicos (15,8\%); do sistema osteomuscular e tecido conjuntivo (9,2\%); transtornos mentais e comportamentais $(7,9 \%)$ e do aparelho respiratório (7,6\%). Quanto ao eixo utilização dos serviços de saúde, foi identificado que a maioria dos usuários referiu apenas um objetivo do comparecimento à UBS $(94,5 \%)$, sendo os mais frequentes consulta médica $(45,0 \%)$; acompanhar atendimento de outras pessoas (30,0\%); consulta com outro profissional de saúde $(7,9 \%)$; agendar consulta/exame para si/terceiros $(5,3 \%)$. Outros motivos totalizaram $12,4 \%$ (em ordem decrescente de frequência: aferir pressão arterial ou glicemia; curativo; vacina; cadastrar-se na UBS ou Cartão Nacional do SUS; buscar medicamentos ou insumos para saúde; buscar informações de saúde e buscar documentação). Dentre os usuários que acompanhavam o atendimento de outras pessoas $(\mathrm{n}=112), 79,5 \%$ estavam com os filhos, sobrinhos ou netos. Verificou-se mediana de 30 (DP 343,9) dias decorridos desde o último comparecimento à UBS (mínimo 1 e máximo 5.475 dias).

A Tabela 2 apresenta as medidas de tendência central e dispersão dos domínios do WHOQOL. Observou-se que os domínios psicológico e relações sociais foram os que apresentaram as maiores médias (respectivamente, 72,25 e 73,59). Estes achados indicaram melhor qualidade de vida dos entrevistados em relação a esses domínios quando comparados ao físico e ao meio ambiente, ambos com escores médios inferiores a 70,00.

Tabela 2

Medidas de tendência central e dispersão dos domínios do WHOQOL breve. Belo Horizonte, MG, 2013. (n=380)

\begin{tabular}{|c|c|c|c|c|c|}
\hline Domínios & Média & $\begin{array}{l}\text { Desvio } \\
\text { padrão }\end{array}$ & Mediana & Mínimo & Máximo \\
\hline Físico & 69,26 & 15,95 & 71,43 & 14,29 & 100,00 \\
\hline Psicológico & 72,25 & 13,34 & 75,00 & 20,83 & 100,00 \\
\hline Relações sociais & 73,59 & 15,30 & 75,00 & 16,67 & 100,00 \\
\hline Meio ambiente & 60,12 & 13,45 & 59,38 & 18,75 & 93,75 \\
\hline
\end{tabular}

Nota: WHOQOL - World Health Organization Quality of Life. 
No que se refere ao letramento em saúde avaliado pelo SAHLPA-18, dos 368 participantes que responderam à avaliação de forma completa, 74,5\% $(n=274)$ apresentaram letramento em saúde inadequado e 25,5\% (n=94), adequado. A mediana dos escores do SAHLPA-18 foi de 12,0 (DP 4,1); média 11,3; pontuação mínima $=1,0$ e máxima $=18,0$.

A Tabela 3 apresenta a análise bivariada e a comparação de medianas entre letramento em saúde e características sociodemográficas. Foram verificadas associações, ao nível de $20 \%$ ( $\mathrm{p} \leq 0,20)$, entre o letramento em saúde e as variáveis sociodemográficas: sexo, escolaridade (em nível de ensino formal), CCEB, plano de saúde, estudo formal mínimo em anos e arranjo familiar.

Tabela 3

Análise de associação (bivariada e comparação de medianas) entre o letramento em saúde e características sociodemográficas. Belo Horizonte, MG, 2013. $(n=368)^{*}$

\begin{tabular}{|c|c|c|c|}
\hline \multirow{2}{*}{ Características } & \multicolumn{2}{|c|}{ Letramento em saúde } & \multirow{2}{*}{ Valor de $\mathrm{p}$} \\
\hline & Adequado & Inadequado & \\
\hline Sexo & n (\%) & n (\%) & \\
\hline Feminino & $58(61,7)$ & $133(48,5)$ & \\
\hline Masculino & $36(38,3)$ & $141(51,5)$ & $0,028 * *$ \\
\hline Total & $94(100,0)$ & $274(100,0)$ & \\
\hline \multicolumn{4}{|l|}{ Situação conjugal } \\
\hline Solteiro & $22(23,4)$ & $89(32,5)$ & \multirow{5}{*}{$0,224 * *$} \\
\hline Casado/União estável & $61(64,9)$ & $152(55,5)$ & \\
\hline & & & \\
\hline Separado/Divorciado/Viúvo & $11(11,7)$ & $33(12,0)$ & \\
\hline Total & $94(100,0)$ & $274(100,0)$ & \\
\hline \multicolumn{4}{|c|}{ Escolaridade (em nível de ensino formal) } \\
\hline Fundamental & $12(12,8)$ & $142(51,8)$ & \multirow{4}{*}{$<0,001 * *$} \\
\hline Médio & $53(56,4)$ & $117(42,7)$ & \\
\hline Superior & $29(30,9)$ & $15(5,5)$ & \\
\hline Total & $94(100,0)$ & $274(100,0)$ & \\
\hline \multicolumn{4}{|l|}{ Situação da moradia } \\
\hline Casa própria & $67(71,3)$ & $180(65,9)$ & \multirow{3}{*}{$0,341^{* *}$} \\
\hline Casa alugada/cedida & $27(28,7)$ & $93(34,1)$ & \\
\hline Total & $94(100,0)$ & $274(100,0)$ & \\
\hline \multicolumn{4}{|c|}{ Critério de Classificação Econômica Brasil } \\
\hline Classes A e B & $39(41,5)$ & $85(32,0)$ & \multirow{5}{*}{$0,033 * *$} \\
\hline Classe C & $52(55,3)$ & $151(56,8)$ & \\
\hline & & & \\
\hline Classes D e E & $3(3,2)$ & $30(11,3)$ & \\
\hline Total & $94(100,0)$ & $266(100,0)$ & \\
\hline
\end{tabular}




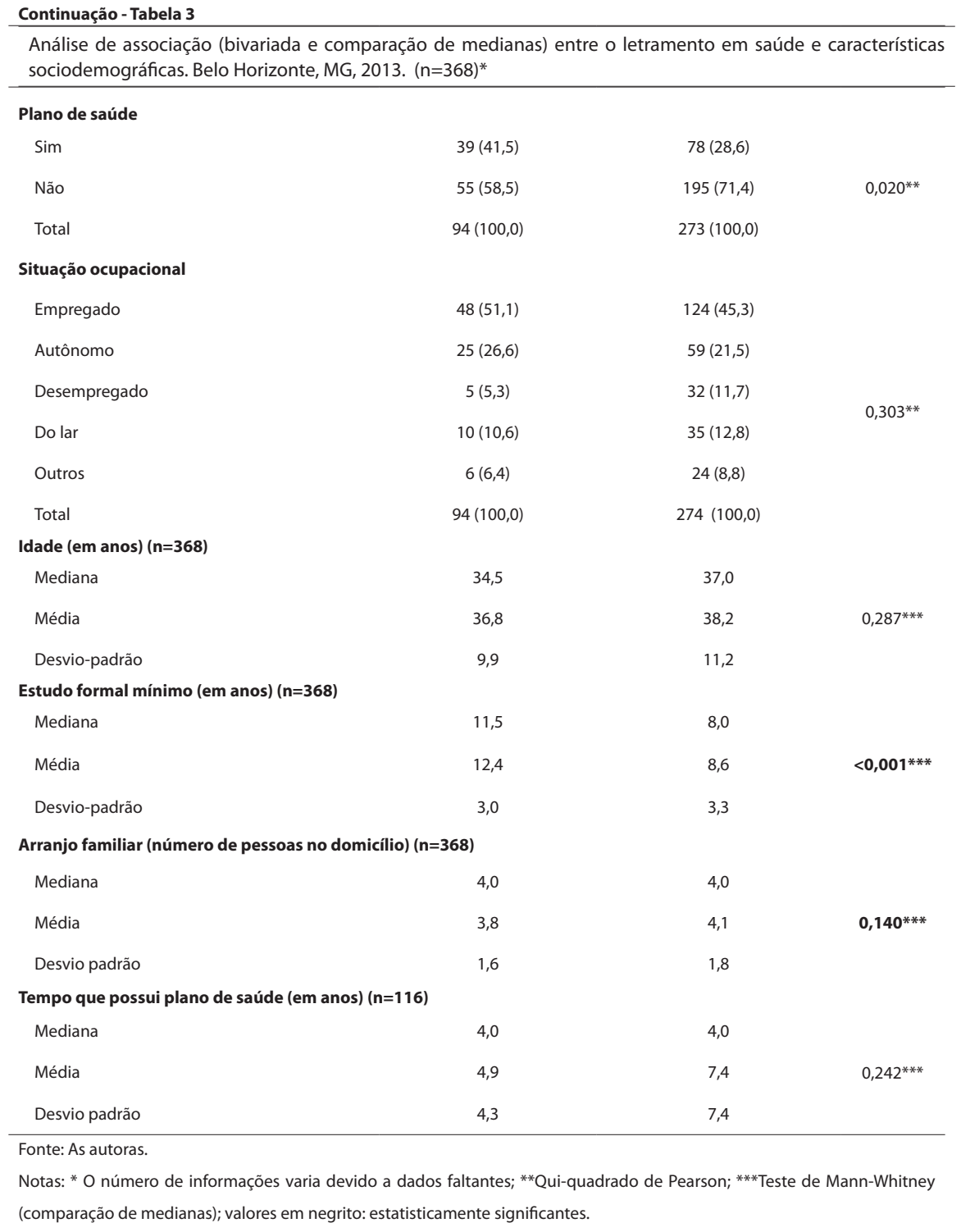

Na Tabela 4, verifica-se a análise de associação (bivariada e comparação de medianas) entre o letramento em saúde e a autopercepção da saúde. Foram observadas associações, ao nível de $20 \%$ ( $\mathrm{p} \leq 0,20)$, entre o letramento em saúde e as seguintes variáveis de autopercepção da saúde: importância atribuída à saúde, nota atribuída à própria saúde e frequência de comparecimento à UBS. 
Tabela 4

Análise de associação (bivariada e comparação de medianas) entre o letramento em saúde e a autopercepção da saúde. Belo Horizonte, MG, 2013. ( $n=368)^{*}$

\begin{tabular}{|c|c|c|c|}
\hline \multirow{2}{*}{ Autopercepção da saúde } & \multicolumn{2}{|c|}{ Letramento em saúde } & \multirow{2}{*}{ Valor de $p$} \\
\hline & Adequado & Inadequado & \\
\hline Importância atribuída à saúde & $n(\%)$ & n (\%) & \\
\hline Sem importância/Pouco importante/Indiferente & $3(3,2)$ & $5(1,8)$ & \\
\hline Importante & $5(5,3)$ & $44(16,1)$ & \\
\hline Muito importante & $86(91,5)$ & $225(82,1)$ & $0,025^{* *}$ \\
\hline Total & $94(100,0)$ & $274(100,0)$ & \\
\hline \multicolumn{4}{|l|}{ Frequência em que pensa na saúde } \\
\hline Nunca/Raramente & $6(6,4)$ & $16(5,8)$ & \\
\hline Às vezes & $15(16,0)$ & $68(24,8)$ & \\
\hline Frequentemente & $33(35,1)$ & $71(25,9)$ & $0,206^{* *}$ \\
\hline Sempre & $40(42,6)$ & $119(43,4)$ & \\
\hline Total & $94(100,0)$ & $274(100,0)$ & \\
\hline \multicolumn{4}{|l|}{ Frequência em que pensa nos problemas de saúde } \\
\hline Nunca/Raramente & $8(8,6)$ & $33(12,1)$ & \\
\hline Às vezes & $25(26,9)$ & $63(23,1)$ & \\
\hline Frequentemente & $26(28,0)$ & $70(25,6)$ & $0,697^{* *}$ \\
\hline Sempre & $34(36,6)$ & $107(39,2)$ & \\
\hline Total & $93(100,0)$ & $273(100,0)$ & \\
\hline
\end{tabular}

Frequência em que o estado de saúde atual compromete as atividades cotidianas

\begin{tabular}{|c|c|c|c|}
\hline Nunca & $52(55,3)$ & $158(57,9)$ & \multirow{6}{*}{$0,915^{* *}$} \\
\hline Raramente & $10(10,6)$ & $24(8,8)$ & \\
\hline Às vezes & $20(21,3)$ & $58(21,2)$ & \\
\hline Frequentemente & $7(7,4)$ & $15(5,5)$ & \\
\hline Sempre & $5(5,3)$ & $18(6,6)$ & \\
\hline Total & $94(100,0)$ & $273(100,0)$ & \\
\hline \multicolumn{4}{|c|}{ Considera-se saudável } \\
\hline Sim & $74(78,7)$ & $219(81,1)$ & \\
\hline Não & $20(21,3)$ & $51(18,9)$ & $0,615^{* * *}$ \\
\hline Total & $94(100,0)$ & $270(100,0)$ & \\
\hline \multicolumn{4}{|c|}{ Avaliação da própria saúde } \\
\hline Ruim & $14(14,9)$ & $44(16,1)$ & \\
\hline Boa & $80(85,1)$ & $230(83,9)$ & $0,789^{* * *}$ \\
\hline Total & $94(100,0)$ & $274(100,0)$ & \\
\hline
\end{tabular}


Análise de associação (bivariada e comparação de medianas) entre o letramento em saúde e a autopercepção da saúde. Belo Horizonte, MG, 2013. $(n=368)^{*}$

Nota atribuída à própria saúde

\begin{tabular}{|c|c|c|c|}
\hline Mediana & 8,0 & 8,0 & \\
\hline Média & 8,0 & 8,1 & $0,142 * * *$ \\
\hline Desvio padrão & 1,2 & 1,7 & \\
\hline
\end{tabular}

Relato de problema de saúde

\begin{tabular}{|c|c|c|c|}
\hline Sim & $51(54,3)$ & $144(52,6)$ & \\
\hline Não & $43(45,7)$ & $130(47,4)$ & $0,776^{* *}$ \\
\hline Total & $94(100,0)$ & $274(100,0)$ & \\
\hline \multicolumn{4}{|c|}{ Número de problemas de saúde relatados } \\
\hline$\leq 1$ & $76(80,9)$ & $223(81,4)$ & \\
\hline$>1$ & $18(19,1)$ & $51(18,6)$ & $0,341^{* *}$ \\
\hline Total & $94(100,0)$ & $274(100,0)$ & \\
\hline
\end{tabular}

Frequência de comparecimento à UBS

\begin{tabular}{|c|c|c|c|}
\hline Nunca/Raramente & $5(7,9)$ & $29(18,8)$ & \\
\hline Às vezes & $17(27,0)$ & $51(33,1)$ & \\
\hline Frequentemente & $23(36,5)$ & $40(26,0)$ & $0,093 * *$ \\
\hline Sempre & $18(28,6)$ & $34(22,1)$ & \\
\hline Total & $63(100,0)$ & $154(100,0)$ & \\
\hline \multicolumn{4}{|c|}{ Tempo decorrido desde a última visita à UBS (em dias) } \\
\hline Mediana & 30,0 & 30,0 & \\
\hline Média & 76,0 & 114,9 & $0,496^{* * *}$ \\
\hline Desvio padrão & 109,0 & 232,0 & \\
\hline
\end{tabular}

Fonte: As autoras.

Notas: * O número de informações varia devido a dados faltantes; **Qui-quadrado de Pearson; ***Teste de Mann-Whitney; UBS: unidade básica de saúde; valores em negrito: estatisticamente significantes.

A análise de associação pela comparação de medianas entre o letramento em saúde e os domínios da qualidade de vida é apresentada na Tabela 5. Os resultados demonstraram que houve associação estatística, ao nível de $20 \%$ $(\mathrm{p} \leq 0,20)$, da variável resposta letramento em saúde com os domínios relações sociais e meio ambiente do WHOQOL. 
Tabela 5

Comparação de medianas entre letramento em saúde e domínios da qualidade de vida. Belo Horizonte, MG, 2013. $(n=368)$

\begin{tabular}{|c|c|c|c|c|c|c|c|}
\hline \multirow{2}{*}{$\begin{array}{l}\text { Qualidade de } \\
\text { vida }\end{array}$} & \multicolumn{2}{|c|}{ Letramento em saúde } & \multirow{2}{*}{$\begin{array}{l}\text { Valor } \\
\text { de } p^{*}\end{array}$} & \multirow{2}{*}{$\begin{array}{c}\text { Qualidade } \\
\text { de vida }\end{array}$} & \multicolumn{2}{|c|}{ Letramento em saúde } & \multirow{2}{*}{$\begin{array}{l}\text { Valor } \\
\text { de } p^{*}\end{array}$} \\
\hline & Adequado & Inadequado & & & Adequado & Inadequado & \\
\hline \multicolumn{4}{|c|}{ Domínio físico } & \multicolumn{3}{|c|}{ Domínio relações sociais } & \\
\hline Mediana & 71,4 & 71,4 & & Mediana & 75,0 & 75,0 & \\
\hline Média & 68,4 & 69,6 & 0,486 & Média & 75,9 & 72,6 & 0,161 \\
\hline Desvio padrão & 16,1 & 15,9 & & Desvio padrão & 13,9 & 15,7 & \\
\hline \multicolumn{4}{|c|}{ Domínio psicológico } & \multicolumn{4}{|c|}{ Domínio meio ambiente } \\
\hline Mediana & 75,0 & 75,0 & & Mediana & 62,5 & 59,4 & \\
\hline Média & 72,9 & 72,1 & 0,642 & Média & 62,0 & 59,5 & 0,149 \\
\hline Desvio padrão & 13,0 & 13,5 & & Desvio padrão & 12,0 & 13,9 & \\
\hline
\end{tabular}

Fonte: As autoras.

Nota: *Teste de Mann Whitney.

Os resultados da análise de regressão logística múltipla hierarquizada dos fatores associados ao letramento em saúde inadequado estão na Tabela 6 . Os achados demonstraram que no modelo final da regressão logística apenas a variável categórica escolaridade (em nível de ensino formal) manteve-se associada ao letramento em saúde de forma estatisticamente significante ao nível de $5 \%(p \leq 0,05)$. Desse modo, verificou-se que os adultos com ensino fundamental e médio demonstravam, respectivamente, 22,06 e 4,20 vezes mais chances de apresentar o letramento em saúde inadequado quando comparados àqueles com ensino superior. 
Tabela 6

Fatores associados ao letramento em saúde inadequado segundo análise de regressão logística múltipla hierarquizada. Belo Horizonte, MG, 2013.

\begin{tabular}{|c|c|c|c|c|}
\hline \multirow{2}{*}{ Características* } & \multicolumn{2}{|c|}{ Modelo inicial } & \multicolumn{2}{|c|}{ Modelo final } \\
\hline & OR (IC 95\%) & Valor de $\mathrm{p}^{*}$ & OR (IC 95\%) & Valor de $\mathbf{p}^{*}$ \\
\hline Sexo & $1,66(0,76-3,59)$ & 0,203 & $\begin{array}{c}1,52(0,90- \\
2,58)\end{array}$ & 0,117 \\
\hline \multicolumn{5}{|l|}{ Escolaridade } \\
\hline Ensino fundamental & $38,68(10,1-148,81)$ & $<0,001$ & $\begin{array}{c}22,06(9,34- \\
52,17)\end{array}$ & $<0,001$ \\
\hline Ensino médio & $4,66(1,71-12,66)$ & 0,003 & $\begin{array}{c}4,20(2,07- \\
8,51)\end{array}$ & $<0,001$ \\
\hline \multicolumn{5}{|l|}{ CCEB } \\
\hline Classes $\mathrm{C} 1 / \mathrm{C} 2$ & $0,56(0,23-1,36)$ & 0,200 & - & - \\
\hline Classes D/E & $1,35(0,13-14,66)$ & 0,803 & - & - \\
\hline Plano de saúde (não) & $1,23(0,55-2,78)$ & 0,616 & - & - \\
\hline Arranjo familiar & $1,06(0,85-1,35)$ & 0,588 & - & - \\
\hline Importância da saúde & $2,96(0,84-10,43)$ & 0,092 & - & - \\
\hline Sem importância & - & - & & \\
\hline Importante & $2,54(0,72-9,01)$ & 0,148 & - & - \\
\hline \multicolumn{5}{|l|}{ Frequência UBS } \\
\hline Nunca/Raramente & $1,91(0,51-7,15)$ & 0,336 & - & - \\
\hline Às vezes & $2,25(0,84-6,07)$ & 0,109 & - & - \\
\hline Frequentemente & $0,92(0,36-2,34)$ & 0,864 & - & - \\
\hline Nota saúde & $0,937(0,69-1,28)$ & 0,678 & & \\
\hline Domínio relações sociais & $1,07(0,98-1,04)$ & 0,646 & - & - \\
\hline Domínio meio ambiente & $0,99(0,97-1,03)$ & 0,962 & - & - \\
\hline Constante & 0,206 & 0,348 & 0,437 & 0,014 \\
\hline
\end{tabular}

Fonte: As autoras.

Nota: Categorias de referência: sexo - masculino; ensino superior; CCEB (Critério de Classificação Econômica Brasil) - classes A/B1/B2; plano de saúde - sim; importância da saúde - muito importante; frequência com que comparece à unidade Básica de Saúde (UBS) - sempre.

Ajuste dos modelos inicial/final (Hosmer e Lemeshow): $p=0,762 / p=0,971$.

\section{Panorama dos estudos científicos em relação ao letramento em saúde e fatores associados}

O letramento em saúde inadequado foi frequente e, na análise bivariada, associado às variáveis sexo, escolaridade, última série cursada com aprovação, estudo formal mínimo, CCEB, plano de saúde, arranjo familiar, importância atribuída à saúde, nota atribuída à própria saúde, frequência de comparecimento à UBS e aos domínios relações sociais e meio ambiente do WHOQOL. 
Após análise de regressão logística, no modelo final, somente a variável escolaridade permaneceu associada ao letramento em saúde, sendo que indivíduos com ensino fundamental e médio apresentaram, respectivamente, 22,1 e 4,2 vezes a chance de ter o letramento em saúde inadequado quando comparados àqueles com ensino superior.

Observou-se elevada frequência de letramento em saúde inadequado em estudos realizados no Brasil: 58,0\% em pacientes de 19 a 59 anos de um hospital universitário (Coelho et al., 2014); bem como 65,9\% entre diabéticos tipo 2 (19 a 59 anos) atendidos em hospital público (Sampaio et al., 2015) (em ambos os estudos a avaliação foi feita por meio do Brief Test of Functional Health Literacy - B-TOFHLA) e 66\% entre idosos (idade $\geq 60$ anos) de clínicas geriátricas públicas avaliados com o SAHLPA-50 (Apolinario et al., 2012). De modo similar, porém mais expressivo, grande percentual dos adultos avaliados no estudo também apresentou letramento em saúde inadequado. A diversidade de métodos das pesquisas, como os diferentes testes utilizados, a aplicação do SAHLPA-18 em ambiente de sala de espera e com amostra de idade diferente em relação à utilizada pelos autores do SAHLPA-18 (Apolinario et al., 2012) podem ser a razão dos diferentes percentuais.

Verificou-se que o letramento em saúde adequado foi mais frequente entre as mulheres, todavia a associação da variável sexo com o letramento em saúde não foi demonstrada no modelo final da regressão logística. Outros estudos na literatura também não identificaram associação entre sexo e letramento em saúde (Apolinario et al., 2012; Sampaio et al., 2015). Entretanto, estudo constatou que a mulher é a principal cuidadora no âmbito das famílias. Ressaltou também que as habilidades comunicativas das mulheres favorecem o desenvolvimento de redes de contato e apoio que possibilitam cuidar com mais eficiência. Esses cuidados abrangem acompanhamento das ações e prescrições médicas (como marcação e acompanhamento das consultas e obtenção de medicamentos) (Gutierrez e Minayo, 2009). Tais achados sugerem que as funções exercidas pelas mulheres e a frequência de comparecimento aos serviços de saúde podem contribuir para o melhor desempenho nas habilidades de letramento em saúde, apesar de esse fato não ter se confirmado no estudo aqui apresentado.

A escolaridade demonstrou importante associação com o letramento em saúde no modelo final da regressão logística, de maneira que quanto maior a escolaridade, maior a frequência de letramento em saúde adequado. Resultados semelhantes foram identificados em outras pesquisas nas quais menos anos de estudo formal estavam associados a pior letramento em saúde (Sampaio et al., 2015) e ensino médio completo associou-se a melhores escores de letramento em saúde, em comparação com indivíduos com menor nível educacional (Beauchamp et al., 2015). Esses achados sugerem que apesar de o letramento em saúde e a escolaridade serem medidas distintas (Apolinário 
et al., 2012; Sampaio et al., 2015), ações que favoreçam a escolaridade formal podem contribuir para reduzir a prevalência de letramento em saúde inadequado. Todavia, cabe ressaltar que o alfabetismo pleno (o qual se espera a partir da conclusão do ensino fundamental) foi alcançado por apenas $35 \%$ das pessoas com ensino médio e $62 \%$ daquelas com ensino superior, de acordo com o Indicador de Alfabetismo Funcional (Inaf) 2011-2012 (Instituto Paulo Montenegro, 2012), que avalia brasileiros de 15 a 64 anos. Tais resultados demonstram que esforços para aprimorar a qualidade do ensino devem ser concomitantes aos avanços já alcançados quanto ao maior acesso à educação formal e mais anos de escolaridade. Portanto, os serviços de saúde devem estar atentos aos indivíduos com menor escolaridade, pois há maiores chances de que estes apresentem limitações quanto ao letramento em saúde, porém é necessário o alerta de que pessoas com níveis mais elevados de ensino formal também podem apresentar essas dificuldades.

A classificação econômica mostrou-se associada ao letramento em saúde na análise bivariada, mas isso não se confirmou no modelo final da regressão logística. Não foram encontrados estudos brasileiros que analisassem essa associação para comparação. Todavia, a literatura internacional refere que o letramento em saúde mais baixo é mais prevalente em grupos populacionais de baixa renda (World Health Organization, 2013).

Verificou-se que a maioria dos participantes não tinha planos de saúde privados, e a posse desses planos esteve associada ao letramento em saúde adequado ao nível p $\leq 0,20$, na análise bivariada. Essa associação também esteve presente em estudo que avaliou 813 adultos de 19 a 99 anos na Austrália (Beauchamp et al., 2015). Todavia, no estudo aqui apresentado, essa associação não se comprovou no modelo final da regressão logística, possivelmente pelo fato de a presença de plano de saúde privado estar relacionada a outros fatores, como a maior escolaridade.

Quanto ao arranjo familiar, na literatura internacional constatou-se que morar sozinho estava associado a piores escores de letramento em saúde, todavia o tamanho do efeito dessa associação foi pequeno (Beauchamp et al., 2015). De modo similar, na pesquisa que originou este artigo o arranjo familiar demonstrou associação com o letramento em saúde somente na análise bivariada, fato que não se confirmou na regressão logística.

No eixo autopercepção da saúde, a importância atribuída à saúde e a nota atribuída à própria saúde demonstraram associação com o letramento em saúde apenas na análise bivariada e não no modelo final da regressão logística. Não foram encontradas na literatura estudos que analisassem a associação entre essas variáveis. Contudo, pesquisa americana constatou que o baixo letramento em saúde, individual e comunitário, está associado à autopercepção de saúde ruim (Sentell et al., 2013). 
Observou-se que os participantes com letramento em saúde adequado relataram de forma mais recorrente comparecer às UBSs sempre ou frequentemente quando comparados àqueles com LS inadequado. Mas essa associação não se confirmou na regressão logística, e não foram identificados outros estudos na literatura que tenham realizado análise semelhante.

Quanto ao eixo qualidade de vida, os domínios relações sociais e meio ambiente do WHOQOL foram associados ao letramento em saúde na análise bivariada, mas não no modelo final da regressão logística. Não foram verificados estudos nacionais que avaliassem essa associação. Os resultados do estudo aqui apresentado divergiram de pesquisa canadense na qual observou-se que o letramento em saúde inadequado estava associado a pior qualidade de vida relacionada à saúde em adultos com diabetes tipo 2 (Sayah, Qiu e Johnson, 2015). A diversidade de aspectos socioeconômicos e culturais entre as populações estudadas possivelmente justificam as diferenças nos achados.

Ressalta-se que os achados do presente estudo restringem-se ao nível funcional do letramento em saúde e são generalizáveis apenas para o município analisado. Contudo, é possível que realidade semelhante seja verificada em outras regiões, uma vez que há similaridade da distribuição em classes econômicas dessa pesquisa, quando comparada aos valores estimados para o Brasil segundo os parâmetros do CCEB a partir de 2015. Em ambos os casos há predomínio das classes C e B (respectivamente) (Associação Brasileira de Pesquisadores de Mercado, Opinião e Mídia, 2015). Assim, acredita-se que a pesquisa tenha contribuído para a identificação do perfil de letramento em saúde de adultos usuários da atenção primária à saúde no SUS. Além disso, a análise dos fatores associados ao letramento em saúde inadequado, tendo como referência a diversidade de variáveis utilizadas nesse estudo, pode direcionar o planejamento de intervenções em saúde. Tais resultados sugerem que são necessárias ações de educação em saúde direcionadas para adultos com todos os níveis de escolaridade, uma vez que o letramento em saúde inadequado é frequente, mas principalmente para os indivíduos com menor escolaridade.

Destaca-se também a necessidade de redefinir estratégias para aprimorar o acompanhamento longitudinal dos adultos usuários da atenção primária no SUS, conforme evidenciado na literatura (Dilélio et al., 2015), pois apesar de muitos indivíduos relatarem problemas de saúde, esses adultos às vezes passam longos períodos sem comparecer às UBS, e quando o fazem frequentemente têm o objetivo de acompanhar familiares e não o próprio cuidado. A atenção primária como local privilegiado na rede de atenção à saúde para o desenvolvimento da promoção do bem-estar e prevenção de doenças, assim como os adultos, protagonistas dos cuidados de si mesmos e muitas vezes também responsáveis pelos de seus familiares, precisam encontrar maneiras para que o suporte longitudinal em saúde esteja ao alcance da população e seja eficiente. 
Cabe ressaltar ainda que para o desenvolvimento do letramento em saúde da população é de extrema relevância a qualidade da interação entre profissionais de saúde, sistema de saúde e usuários. Portanto, além das ações de educação em saúde dos usuários, é fundamental incluir o letramento em saúde na formação dos profissionais da área (Passamai et al., 2012), porque apesar da característica interdisciplinar deste tema e da sua importância para o sucesso das intervenções, este permanece um assunto pouco conhecido e discutido na formação e atuação profissional.

Em síntese, o estudo que originou este artigo procurou a hipótese de que a menor escolaridade esteja associada ao letramento em saúde inadequado. Além disso, identificou-se que as demais variáveis sociodemográficas, de autopercepção de saúde e qualidade de vida, não foram associadas ao letramento em saúde quando este foi avaliado mediante o teste SAHLPA-18. A análise dos resultados da pesquisa e da literatura levam a crer na hipótese de que o letramento em saúde dos adultos pode ser aprimorado por meio de ações no âmbito da educação em saúde, do fortalecimento do suporte longitudinal na atenção primária e da autonomia dos usuários das UBSs, da qualidade da interação entre estes usuários e trabalhadores da saúde, e também no contexto da inclusão do tema letramento em saúde na formação e prática dos profissionais de saúde.

\section{Considerações finais}

Dentre os fatores sociodemográficos analisados, a escolaridade foi a única variável que apresentou associação positiva com o letramento em saúde, e indivíduos com menor escolaridade tiveram mais chance de ter o letramento em saúde inadequado. A autopercepção da saúde e a qualidade de vida não foram associadas ao letramento em saúde. Os achados sugerem a necessidade de se desenvolverem ações de educação em saúde direcionadas para os adultos usuários da atenção primária e com a participação desses indivíduos, a fim de que se alcance o acompanhamento longitudinal da saúde de forma eficiente.

\section{Colaboradores}

Suzana Raquel Lopes Marques responsabilizou-se pela elaboração da pesquisa e coleta de dados, redação e formatação do artigo; Stela Maris Aguiar Lemos, pela elaboração da pesquisa, orientação e revisão crítica do artigo. Não houve financiamento para a pesquisa e não há conflitos de interesse. 
Resumen El objeto fue investigar la alfabetización en salud y asociación con factores sociodemográficos, autopercepción de salud y calidad de vida. Este es un estudio transversal de adultos entre 20 y 59 años, usuarios de atención primaria de salud, que se realizó en unidades básicas de salud, de febrero a mayo de 2015 - muestra de probabilidad. La alfabetización en salud se evaluó mediante el Short Assessment of Health Literacy for Portuguese-speaking Adults. Un análisis estadístico descriptivo, análisis bivariado $(\mathrm{p} \leq 0,20)$ y regresión logística múltiple $(\mathrm{p} \leq 0,05)$ fue realizada. La alfabetización en salud inadecuada fue frecuente y en principio asociada con sexo, escolaridad, grado más alto con aprobación, estudio formal mínimo, Criterio de Clasificación Económica Brasil, seguro de salud, estructura familiar, importancia asignada a propia salud, puntuación para propia salud, frecuencia en la unidade básica de salud y dominios relaciones sociales y ambiental de la prueba World Health Organization Quality of Life. En el modelo final de regresión logística, sólo escolaridad se mantuvo asociado con alfabetización en salud, de modo que personas con menos escolaridad eran más propensas a tener alfabetización en salud inadecuada. Los hallazgos sugieren necesidad de desarrollar estrategias de educación en salud para adultos de atención primaria.

Palabras clave alfabetización en salud; condiciones sociales; educación en salud; salud del adulto; sistema único de salud.

\section{Notas}

${ }^{1}$ Universidade Federal de Minas Gerais, Programa de Pós-Graduação em Ciências Fonoaudiológicas, Belo Horizonte, Minas Gerais, Brasil.

<suzilps@yahoo.com.br>

Correspondência: Rua Dr. Luís Ratton, 108, apto. 202/bl. 1, CEP 30840-600, Manacás, Belo Horizonte, Minas Gerais, Brasil.

${ }^{2}$ Universidade Federal de Minas Gerais, Programa de Pós-Graduação em Ciências Fonoaudiológicas, Belo Horizonte, Minas Gerais, Brasil.

$<$ lemos.stela@gmail.com>

${ }^{3}$ Este artigo é resultado de dissertação de mestrado em Ciências Fonoaudiológicas, da Universidade Federal de Minas Gerais, intitulada Letramento em saúde: associação com dados sociodemográficos, autopercepção de saúde e qualidade de vida em adultos usuários da atenção primária à saúde, de autoria de Marques (2016).

${ }^{4}$ As referências bibliográficas relativas ao instrumento SAHLPA-18 são baseadas em Apolinario et al., 2012. Nesta referência, na página 704, item “SAHLPA Administration and Scoring", o autor se dispõe a enviar aos interessados o material para aplicação do teste: "User instructions and laminated card sets are available upon request from the corresponding author." Esse material de aplicação do SAHLPA-18 foi então por nós solicitado ao autor, que nos enviou por mensagem eletrônica pessoal, tendo sido utilizado no trabalho aqui apresentado, na seção "Critérios e instrumentos para avaliação do letramento em saúde e das variáveis explicativas". 


\section{Referências}

AGOSTINHO, Milena R. et al. A autopercepção da saúde entre usuários da atenção primária em Porto Alegre, RS. Revista Brasileira de Medicina de Família e Comunidade, Porto Alegre, v. 5, n. 17, p. 9-15, 2010.

APOLINARIO, Daniel et al. Short assessment of health literacy for Portuguese-speaking adults. Revista de Saúde Pública, São Paulo, v. 46, n. 4, p. 702-711, 2012.

AQUINO, Estela M. L. et al. Brazilian Longitudinal Study of Adult Health (ELSA-Brasil): objectives and design. American Journal of Epidemiology, Salvador, v. 175, n. 4, p. 315324, 2012.

\section{ASSOCIAÇÃO BRASILEIRA DE EMPRESAS} DE PESQUISA (ABEP). Critério de Classificação Econômica Brasil - 2012. Dados com base no levantamento socioeconômico 2010 - Ibope. Disponível em: < file://C:/Users/Suzana/ Downloads/03_cceb_2012_base_lse_2010. pdf > Acesso em: 9 set. 2013.

\section{ASSOCIAÇÃO BRASILEIRA DE PESQUISA-} DORES DE MERCADO, OPINIÃO E MÍDIA (ASBPM). Alterações na aplicação do Critério Brasil, válidas a partir de 01/01/2015. Disponível em: <http://www.asbpm.org.br/criterios. asp>. Acesso em: 12 mar. 2016.

AZEVEDO, Gezaine P. G. C.; FRICHE, Amélia A. L.; LEMOS, Stela M. A. Autopercepção de saúde e qualidade de vida de usuários de um ambulatório de fonoaudiologia. Revista da Sociedade Brasileira de Fonoaudiologia, São Paulo, v. 17, n. 2, p. 119-127, 2012.

BEAUCHAMP, Alison et al. Distribution of health literacy strengths and weaknesses across socio-demographic groups: a cross-sectional survey using the Health Literacy Questionnaire (HLQ). BioMed Central Public Health, Melbourn, v. 15, p. 678-691, 2015.
CHIESA, Anna M. et al. Possibilidades do WHOQOL-bref para a promoção da saúde na Estratégia Saúde da Família. Revista da Escola de Enfermagem da USP, São Paulo, v. 45, n. Esp. 2, p. 1.743-1.747, 2011.

COELHO, Maria A. M. et al. Functional health literacy and healthy eating: understanding the Brazilian food guide recommendations. Revista de Nutrição, Campinas, v. 27, n. 6, p. 715-723, 2014.

CONFORTIN, Susana C. et al. Autopercepção positiva de saúde em idosos: estudo populacional no Sul do Brasil. Cadernos de Saúde Pública, Rio de Janeiro, v. 31, n. 5, p. 1.0491.060, 2015.

CUNHA, Elenice M.; GIOVANELLA, Ligia. Longitudinalidade/continuidade do cuidado: identificando dimensões e variáveis para a avaliação da atenção primária no contexto do sistema público de saúde brasileiro. Ciência \& Saúde Coletiva, Rio de Janeiro, v. 16, n. 1, p. 1.029-1.042, 2011.

DILÉLIO, Alitéia S. et al. Lack of access and continuity of adult health care: a national population-based survey. Revista de Saúde Pública, São Paulo, v. 49, n. 3, p. 1-15, 2015.

FLECK, Marcelo P. A. et al. Aplicação da versão em português do instrumento abreviado de avaliação da qualidade de vida 'WHOQOLbref'. Revista de Saúde Pública, São Paulo, v. 34, n. 2, p. 178-183, 2000.

GUTIERREZ, Denise M. D.; MINAYO, Maria C. S. Papel da mulher de camadas populares de Manaus na produção de cuidados da saúde. Saúde e Sociedade, São Paulo, v. 18, n. 4, p. 707-720, 2009.

INSTITUTO PAULO MONTENEGRO. Indicador de Alfabetismo Funcional - Brasil 
2011. Principais resultados. 2012. Disponível em: <https://drive.google.com/file/ d/0B5WoZxXFQTCRWE5UY2FiMzFhZEk/ view> Acesso em: 12 mar. 2016.

KESSARAM, Tara et al. Noncommunicable diseases and risk factors in adult populations of several Pacific Islands: results from the WHO STEP wise approach to surveillance. Australian and New Zealand Journal of Public Health, New Caledonia, v. 39, n. 4, p. 336343, 2015.

KLUTHCOVSKY, Ana C. G. C.; KLUTHCOVSKY, Fábio A. O WHOQOL-bref, um instrumento para avaliar qualidade de vida: uma revisão sistemática. Revista de Psiquiatria do Rio Grande do Sul, Porto Alegre, v. 31, n. 3, p. 1-12, 2009.

LEE, Shoou-Yih D. et al. Short assessment of health literacy-Spanish and English: a comparable test of health literacy for Spanish and English speakers. Health Services Research, Chapel Hill, v. 45, n. 4, p. 1.105-1.120, 2010.

MACINKO, James; HARRIS, Matthew J. Brazil's Family Health Strategy: delivering community-based primary care in a universal health system. New England Journal of Medicine, Massachusetts, v. 372, n. 23, p. 2.177-2.181, 2015.

MARQUES, Suzana R. L. Letramento em saúde: associação com dados sociodemográficos, autopercepção de saúde e qualidade de vida em adultos usuários da atenção primária à saúde. 2016. 176 f. Dissertação (Mestrado em Ciências Fonoaudiológicas) - Faculdade de Medicina, Universidade Federal de Minas Gerais, Belo Horizonte, 2016.

NOURI, Sarah S.; RUDD, Rima E. Health literacy in the 'oral exchange': an important element of patient-provider communication. Patient Education and Counseling, Boston, v. 98, p. 565-571, 2015.

NUTBEAM, Don. Health literacy as a public health goal: a challenge for contemporary health education and communication strategies into the 21 st century. Health Promotion International, Oxford, v. 15, n. 3, p. 259-267, 2000.

PAIM, Jairnilson et al. The Brazilian health system: history, advances, and challenges. The Lancet, Salvador, v. 377 , n. 9779 , p. $1.778-$ 1.797, 2011.

PASSAMAI, Maria P. B. et al. Letramento funcional em saúde: reflexões e conceitos sobre seu impacto na interação entre usuários, profissionais e sistema de saúde. Interface: Comunicação, Saúde e Educação, Botucatu, v. 16, n. 41, p. 301-314, 2012.

PEDROSO, Bruno et al. Cálculo dos escores e estatística descritiva do WHOQOL-bref através do Microsoft Excel. Revista Brasileira de Qualidade de Vida, Curitiba, v. 2, n. 1, p. 31-36, 2012.

RASELLA, Davide et al. Impact of primary health care on mortality from heart and cerebrovascular diseases in Brazil: a nationwide analysis of longitudinal data. British Medical Journal, Londres, v. 349, p. 4.014-4.024, 2014.

REICHERT, Felipe F.; LOCH, Mathias R.; CAPILHEIRA, Marcelo F. Autopercepção de saúde em adolescentes, adultos e idosos. Ciência \& Saúde Coletiva, Rio de Janeiro, v. 17, n. 12, p. 3.353-3.362, 2012.

RUIZ, Rolando B. et al. Alfabetización en salud en medicina general integral: perspectivas em Santiago de Cuba. Medisan, Santiago de Cuba, v. 13, n. 1, p. 126-140, 2013.

SAMPAIO, Helena A. C. et al. Letramento em saúde de diabéticos tipo 2: fatores associados e controle glicêmico. Ciência \& Saúde Coletiva, Rio de Janeiro, v. 20, n. 3, p. 865-874, 2015.

SAYAH, Fatima; QIU, Weiyu; JOHNSON, Jeffrey A. Health literacy and health-related quality of life in adults with type 2 diabetes: a longitudinal study. Quality of Life Research, Edmonton, v. 25, n. 6, p. 1-8, 2015. Disponível em: <http:// ncbi.nlm.nih.gov/pubmed/26603739>. Acesso em: 20 dez. 2015. 
SENTELL, Tetine et al. The influence of community and individual health literacy on self-reported health status. Journal of General Internal Medicine, Honolulu, v. 29, n. 2, p. 298-304, 2013.

SIQUEIRA, Arminda L.; TIBÚRCIO, Jacqueline D. Estatística na área da saúde: conceitos, metodologia, aplicações e prática computacional. Belo Horizonte: Coopmed, 2011.

WORLD HEALTH ORGANIZATION (WHO). Programme on mental health: WHOQOL user manual. WHO, 1998, edition 2012.
Disponível em: <http:/apps.who.int/iris/ bitstream/10665/77932/1/WHO_HIS_HSI_ Rev.2012.03_eng.pdf?ua=1>. Acesso em: 12 maio 2014.

WORLD HEALTH ORGANIZATION (WHO). Regional Office for Europe. Health literacy: the solid facts. 2013. Disponível em: < htttp://euro. who.int/_data/assets/pdf_file/0008/190655/ e96854.pdf>.

YIN, Shonna H. et al. Health literacy: an educationally sensitive patient outcome. Journal of General Internal Medicine, New York, v. 30, n. 9 , p. 1.363-1.368, 2015.

Recebido em 22/07/2016. Aprovado em 10/07/2017. 